\title{
ON CERTAIN REPRESENTATIONS OF THE MEASURE ALGEBRA OF A LOCALLY COMPACT ABELIAN GROUP
}

\author{
BY \\ HORST LEPTIN
}

Throughout this paper let $G$ be a locally compact abelian group. Furthermore, let $\mathscr{B}$, resp. $\mathscr{C}_{0}$, resp. $\mathscr{C}_{\infty}$, always be the Banach spaces of all bounded complex Borel functions, resp. bounded continuous functions, resp. continuous functions, which vanish at $\infty$. Then we have $\mathscr{C}_{\infty} \subset \mathscr{C}_{0} \subset \mathscr{B}, \mathscr{C}_{\infty}=\mathscr{C}_{0}$ iff $G$ is compact and $\mathscr{C}_{0}=\mathscr{B}$ iff $G$ is discrete. Every continuous complex linear functional on $\mathscr{C}_{\infty}$ defines a bounded Radon measure on $G$ and thus the set $\mathfrak{M}$ of all bounded Radon measures is the dual space of $\mathscr{C}_{\infty}$. For the value of a measure $\mu \in \mathfrak{M}$ for a certain function $f \in \mathscr{C}_{\infty}$ we use the following notations:

$$
\mu(f)=\int f d \mu=\int f(x) d \mu(x)
$$

The norm in $\mathfrak{M}$ is then defined as $|\mu|=\sup _{|f| \leqq 1}|\mu(f)|$ with $|f|=\sup _{G}|f(x)|$. $\mathfrak{M}$ is a Banach algebra under convolution: For $\mu, v \in \mathfrak{M}$, the product $\mu * v$ is defined as

$$
\mu * v(f)=\int f(x+y) d \mu(x) d v(y)
$$

For a complex function $f$ on $G$ let $f^{*}$ be defined as $f^{*}(x)=\overline{f(-x)}$. Then we can define for $\mu \in \mathfrak{M}: \mu^{*}(f)=\overline{\mu\left(f^{*}\right)}$. The mapping $\mu \rightarrow \mu^{*}$ is an isomeric involution on $\mathfrak{M}$. Let $a$ be a point in $G$ and $\varepsilon_{a}$ the corresponding point measure, defined as $\varepsilon_{a}(f)=f(a)$. We have $\varepsilon_{a}^{*}=\varepsilon_{-a}$. For $a=0$ the measure $\varepsilon_{0}=\varepsilon$ is the identity in $\mathfrak{M}$. Let $\mathfrak{L}^{\mathbf{1}}$ be the convolution algebra of all Haar integrable complex functions on $G$. If we identify $g \in \mathfrak{Q}^{1}$ with the Radon measure $\lambda_{g}(f)=\int f(x) g(x) d x$, we may consider $\mathfrak{L}^{1}$ as a norm closed and symmetric ideal in $\mathfrak{M}$. Here "symmetric" means, that with $\lambda_{g} \in \mathfrak{L}^{1}$ always $\lambda_{g}^{*} \subset \mathfrak{L}^{1}$. In fact, we have even $\lambda_{g}^{*}=\lambda_{g^{*}}$.

Every positive $\mu \in \mathfrak{M}$ defines a regular Borel measure on $G$, so that all bounded Borel functions are integrable with respect to $\mu$. So we can uniquely extend every $\mu \in \mathfrak{M}$ to a bounded linear functional on $\mathscr{B}$. For all definitions, results, etc. here mentioned, also for further questions, see, e.g., $[1 ; 2]$ or [3]. 
1. For $f \in \mathscr{B}, \mu \in \mathfrak{M}$ we define

$$
\left(T_{\mu} f\right)(x)=\mu * \varepsilon_{x}(f)=\int_{G} f(x+t) d \mu(t) .
$$

Then $T_{\mu} f$ is a bounded complex function on $G$ and we have

$$
\begin{aligned}
& \left|T_{\mu} f\right|=\sup _{G}\left|T_{\mu} f(x)\right| \leqq\left|\mu * \varepsilon_{x}\right||f|=|\mu| \cdot|f|, \\
& |\mu|=\sup _{|f| \leqq 1}|\mu(f)|=\sup \left|T_{\mu} f(0)\right| \leqq \sup \left|T_{\mu} f\right|=\left|T_{\mu}\right|,
\end{aligned}
$$

hence $|\mu|=\left|T_{\mu}\right|$. We have

(1) $T_{\mu}$ maps $\mathscr{C}_{\infty}$ into $\mathscr{C}_{\infty}, \mathscr{C}_{0}$ into $\mathscr{C}_{0}$ and $\mathscr{B}$ into $\mathscr{B}$.

Because the measures with compact carrier are dense in $\mathfrak{M}$ and $|\mu|=\left|T_{\mu}\right|$ we need prove (1) only for positive $\mu$ with compact carriers. If then $f$ has compact carrier, $T_{\mu} f$ also has compact carrier, if $f$ is continuous, it is uniformly continuous on every compact subset of $G$. From these facts it follows easily that $T_{\mu} \mathscr{C}_{\infty} \subset \mathscr{C}_{\infty}$ and $T_{\mu} \mathscr{C}_{0} \subset \mathscr{C}_{0}$. For every positive Radon measure and every $\operatorname{directed} \operatorname{set}\left({ }^{1}\right)$ of continuous real functions $f$ we have sup $\mu(f)=\mu(\sup f)$ and therefore sup $T_{\mu} f$ $=T_{\mu}(\sup f)$ if $\sup f \in \mathscr{B}$. Therefore $T_{\mu} f$ is lower semi-continuous, if $f$ is lower semi-continuous. Furthermore, we have $T_{\mu}\left(\lim f_{n}\right)=\lim T_{\mu} f_{n}$ if $\left\{f_{n}\right\}$ is a monotonic sequence in $\mathscr{B}$. It follows that $T_{\mu} \mathscr{B} \subset \mathscr{B}$.

Obviously the $T_{\mu}$ commute with the translations $f \rightarrow f_{a}$, where $f_{a}(x)=f(x+a)$. The following proposition yields a measurefree description of the measure algebra:

(2) The mapping $\mu \rightarrow T_{\mu}$ is an isometric*-isomorphism from $\mathfrak{M}$ onto the algebra $\mathscr{A}$ of all bounded operators of $\mathscr{C}_{\infty}$, which commute with all translations $f \rightarrow f_{a}$ of $\mathscr{C}_{\infty}$.

(Here the operator $S^{*}$ for a bounded operator $S$ of $\mathscr{C}_{\infty}$ is defined as $S^{*} f=\left(S f^{*}\right)^{*}$.)

Proof. Clearly $\mu \rightarrow T_{\mu}$ is a linear and norm preserving mapping of $\mathfrak{M}$ into $\mathscr{A}$. For the product we have

$$
\begin{aligned}
T_{\mu v}^{*} f(x) & =\int f(x+t) d \mu * v(t)=\int f(x+u+v) d \mu(u) d v(v) \\
& =\int T_{\mu} f(x+v) d v(v)=T_{v} T_{\mu} f(x)=T_{\mu} T_{v} f(x),
\end{aligned}
$$

i.e., $T_{\mu * v}=T_{\mu} T_{v}$. Also

$$
T_{\mu^{*}} f=\mu^{*} * \varepsilon_{x}(f)=\left(\mu * \varepsilon_{x}\right)^{*}(f)=\left(A \mu * \varepsilon_{x}\right)\left(f^{*}\right)=T_{\mu}\left(f^{*}\right)^{*}=T_{\mu}^{*}(f) .
$$

Let $A \in \mathscr{A}$. Then $\mu(f)=(A f)(0)$ defines a measure $\mu \in \mathfrak{M}$ and because $A$ permutes with the translations, we have

(1) This means that, for each pair of functions $f$ and $g$ in the set, there exists a function $h$ in the set such that $f \leqq h$ and $g \leqq h$ in the natural (pointwise) order. 


$$
T_{\mu} f(x)=\mu * \varepsilon_{-x}(f)=\left(A f_{x}\right)(0)=(A f)_{x}(0)=(A f)(x),
$$

i.e., $T_{\mu} f=A f$ and consequently $T_{\mu}=A$.

2. A satisfactory description of all maximal ideals of the algebra $\mathfrak{M}$ is still an unsolved problem and so any step forward in this direction may be of some interest. In the case of the algebra $\mathfrak{Q}^{1}$ we know, that all maximal ideals or equivalently all homomorphisms of $\mathfrak{L}^{1}$ onto the complex number field $\mathbf{C}$ are given by means of the formula $\Phi_{\chi}(g)=\int \overline{\chi(x)} g(x) d x$, where $\chi$ is a continuous character of $G$. The same formula gives a homomorphism of $\mathfrak{M}$ in $\mathbf{C}$, if we replace $g d x$ by a general measure $\mu$ :

$$
\Phi_{\chi}(\mu)=\int \overline{\chi(x)} d \mu(x)=\mu(\bar{\chi}) .
$$

In this way, as is well known, we may identify the maximal ideal space of $\mathfrak{Q}^{1}$ with the open complement of the hull of $\mathfrak{L}^{1}$ in the maximal ideal space of $\mathfrak{M}$. Furthermore, it is well known $\left({ }^{2}\right)$ that these homomorphisms are just those given by minimal invariant subspaces of $\mathscr{B}$, invariant under the representation $\mu \rightarrow T_{\mu}$ of $\mathfrak{M}$ in $\mathscr{B}$ : Every minimal invariant subspace of $\mathscr{B}$ is of the form $\{a \chi\}, a \in \mathbf{C}$, $\chi$ a continuous character, and each such function space is a minimal invariant subspace of $\mathscr{B}$. Now the question arises, if it is possible to obtain other homomorphisms of $\mathfrak{M}$ via minimal invariant subspaces in other reasonable representation spaces of $\mathfrak{M}$. In this paper we will show that in the cases of the factor spaces $\mathscr{D}=\mathscr{B} / \mathscr{C}_{0}$ and $\mathscr{E}=\mathscr{B} / \mathscr{C}_{\infty}$ the answer is negative. More explicitly we shall prove the following

THEOREM. If the locally compact abelian group $G$ is separable, then the factorspace $\mathscr{D}$ has no proper minimal invariant subspaces with respect to the representation induced by the representation $\mu \rightarrow T_{\mu}$ of the measure algebra $\mathfrak{M}(G)$ in $\mathscr{B}$. If $G$ is not compact, then every minimal invariant subspace of $\mathscr{E}$ is generated by a function $f=\chi g$, product of a continuous character $\chi$ and $a$ continuous bounded function $g$, having the property that for every $a \in G$ the function $\tilde{g}_{\alpha}: x \rightarrow g(a+x)-g(x)$ vanishes at infinity, i.e., lies in $\mathscr{C}_{\infty}$. Conversely, every function of this form $f=\chi g$ generates an invariant minimal subspace modulo $\mathscr{C}_{\infty}$ and the homomorphism $\Phi$, which is defined by this subspace, is identical with $\Phi_{\chi}$.

Call a function $g \in \mathscr{B}$ slowly oscillating at infinity, if $g(a+x)-g(x)$ vanishes at infinity for every fixed $a$. Of course every $g \in \mathscr{C}_{\infty}$ oscillates slowly at infinity, ut there also exist continuous functions not in $\mathscr{C}_{\infty}$, which are slowly oscillating

(2) Recall the fact, that every continous irreducible representation of a complex commutative Banach-algebra with unit is one-dimensional because the factor-algebra modulo the kernel is a field. By Gelfand's and Mazur's theorem this factor-algebra is equal to C. Particularly every proper minimal invariant subspace must have dimension one. 
at infinity. For example, let $G$ be the real line and $h$ be any periodic differentiable function with continuous derivative. Then $g(x)=h\left(\log \left(1+x^{2}\right)\right)$ oscillates slowly at infinity and is in $\mathscr{C}_{\infty}$ if and only if $h$ is zero.

3. Let $\mathscr{A}$ be any $T$-invariant subspace of $\mathscr{B}, T$ the representation $\mu \rightarrow T_{\mu}$ of $\mathfrak{M}$ in $\mathscr{B}$. If the function $f \in \mathscr{B}$ generates modulo $\mathscr{A}$ a minimal invariant subspace $\left({ }^{2}\right)$, there exists a homomorphism $\Phi$ of $\mathfrak{M}$ in $\mathbf{C}$, such that

or more explicitly:

$$
T_{\mu} f \equiv \Phi(\mu) f(\bmod \mathscr{A})
$$

$$
\left(T_{\mu} f\right)(x)=\Phi(\mu) f(x)+H(\mu, x)
$$

where $H(\mu, x)$ is a function in $\mathscr{A}$ for every $\mu \in \mathfrak{M}$. For $\mu=\varepsilon_{y}$ we have $\left(T_{\varepsilon_{y}} f\right)(x)$ $=f(x+y)$ and $\Phi\left(\varepsilon_{y}\right)=\chi(y)$ is a character of $G$. We write

Then we get from (3):

$$
H\left(\varepsilon_{y}, x\right)=H(y, x) \text {. }
$$

$$
f(x+y)=\chi(x) f(y)+H(x, y) .
$$

Now let $\mathscr{A}$ be always in $\mathscr{C}_{0}$, then $H(x, y)$ is continuous in $y$ for every fixed $x$ and from (4) follows immediately:

If $f$ is continuous in a single point, then it is continuous everywhere.

THEOREM 1. Let $G$ be separable. Let $f$ be a bounded Borel-function on $G$, which satisfies identity (4) with a (not necessarily continuous) character $\chi$. If then the function $H(x, y)$ is partially continuous in $y$, then $f$ is continuous.

Proof. We choose a sequence of integrable positive continuous functions $u_{i}$ with $\int u_{i} d x=1$ and $\lim _{i \rightarrow \infty} \int h(x) u_{i}(x) d x=h(0)$ for every bounded measurable function $h$, which is continuous at 0 . Let in general $f_{1} * f_{2}$ denote the convolution of the functions $f_{1}$ and $f_{2}$ :

$$
\left(f_{1} * f_{2}\right)(x)=\int_{G} f_{1}(x-t) f_{2}(t) d t
$$

For every continuous function $h$ on $G$, we then have $\lim _{i \rightarrow \infty}\left(h * u_{i}\right)(x)=h(x)$. We now write $f_{i}(y)=\left(f * u_{i}\right)(y)$ and $H_{i}(x, y)=\left(H(x, \cdot) * u_{i}\right)(y)$. The functions $f_{i}$ are all continuous and bounded and $\lim H_{i}(x, y)=H(x, y)$, because $H(x, \cdot)$ is a continuous function of $y$. Let us assume that the bounded sequence $\left\{f_{i}(0)\right\}$ converges (otherwise we take a suitable subsequence). It follows from (4) for $y=0$ that $\left\{f_{i}(x)\right\}$ then converges for every $x$. But then the bounded function $f(x)$ $=\lim _{i \rightarrow \infty} f_{i}(x)$ is again measurable and satisfies the same identity (4). For the difference $d=f-f$ we then have

$$
d(x+y)=\chi(x) d(y) \text {. }
$$

But then either $d=0$, i.e., $f=f$, or $\chi(x)=d(x) / d(0)$ is measurable, hence con- 
tinuous, and also $d$ is continuous. Now $\tilde{f}$ as a limit of a sequence of continuous functions, according to a well-known theorem, (see e.g. [4, p. 164]), is continuous in at least one point. It follows that $f=\tilde{f}+d$ is also continuous in at least one point and so is continuous everywhere. This proves Theorem 1.

COROLlARY. The factorspace $\mathscr{B} / \mathscr{C}_{0}$ has no minimal invariant subspaces.

We mention that according to a theorem of Rudin [2, p. 230] the kernel of the factor representation of $\mathfrak{M}$ in $\mathscr{D}$ is equal to $\mathfrak{L}^{1}$.

THEOREM 2. If in (4) $H(x, \cdot)$ is in $\mathscr{C}_{\infty}$ for every $x$ and $f$ is not in $\mathscr{C}_{\infty}$, then $\chi$ is continuous and the function $g=\bar{\gamma} f$ is a continuous function, which oscillates slowly at infinity.

Proof. From Theorem 1 it follows that $f$ is continuous. Now assume that $f$ does not vanish at infinity. Then there exists a sequence $\left\{y_{n}\right\}$ in $G$, which converges to infinity and for which $\lim _{n \rightarrow \infty} f\left(y_{n}\right)=\alpha$ exists and is not 0 . From (4) and $\lim H\left(x, y_{n}\right)=0$ then follows $\lim _{n \rightarrow \infty} f\left(x+y_{n}\right)=\chi(x) \alpha$. This shows that $\chi$ must be measurable and hence continuous. Of course $g=\bar{\chi} f$ is also continuous and satisfies the relation

$$
g(x+y)-g(y)=\bar{\gamma}(x+y) H(x, y) \in \mathscr{C}_{\infty}
$$

for every fixed $x$.

The next theorem holds for general locally compact abelian groups.

THEOREM 3. A subspace $(\tilde{f})$ of $\mathscr{B} / \mathscr{C}_{\infty}$ is invariant under the representation $T$ of $\mathfrak{M}$ in $\mathscr{B} / \mathscr{C}_{\infty}$ if and only if the function $f$, representative of $\tilde{f}=f+\mathscr{C}_{\infty}$, is of the form $f=\chi g$, where $\chi$ is a continuous character of $G$ and $g$ is a bounded continous function, which oscillates slowly at infinity. The homomorphism $\Phi$ of $\mathfrak{M}$, corresponding to $(\tilde{f})$, is identical with the homomorphism $\Phi_{y}$, corresponding to the character $\chi$.

We remark that in the separable case the "only if" part of this theorem is an immediate consequence of Theorem 2. For the general case we need a lemma:

(6) A function $f$ on $G$ converges to zero for $x$ converging to infinity, if and only if $\lim _{n \rightarrow \infty} f\left(x_{n}\right)=0$ for every sequence $\left\{x_{n}\right\} \subset G$ with $\lim _{n \rightarrow \infty} x_{n}=\infty$.

We must show only that the condition is sufficient. This is obvious, if $G$ is countable at infinity, i.e., compactly generated. Now let $G_{0}$ be a compactly generated open subgroup of $G$ and $\varepsilon>0$. Let $\left\{x_{n}\right\} \subset G$ be such that $x_{n}-x_{m} \notin G_{0}$ for $n \neq m$. Then obviously $\lim _{n \rightarrow \infty} x_{n}=\infty$. It follows that $|f(x)|<\varepsilon$ on the residue-classes of $G_{0}$ with at most a finite number of exceptions, say $X_{i}=x_{i}+G_{0}$, $i=1, \cdots, n$. Because $X_{i}$ is homeomorphic to $G_{0}$, and is countable at infinity, there exists a compact subset $K_{i} \subset X_{i}$ with $|f(x)|<\varepsilon$ for $x \notin K_{i}$. Then $K=\bigcup_{i=1}^{n} K_{i}$ is again compact and for all $x \notin K$ we have $|f(x)|<\varepsilon$, which shows that $\lim _{x \rightarrow \infty} f(x)=0$. 
Now let $f$ generate an invariant subspace in $\mathscr{B} / \mathscr{C}_{\infty}$. From (4) the following identity follows:

$$
\chi(x) f(y)+H(x, y)=\chi(y) f(x)+H(y, x) .
$$

Now choose a sequence $\left\{y_{i}\right\} \subset G$ with $\lim _{i \rightarrow \infty} y_{i}=\infty$ in such a way that $\lim f\left(y_{i}\right)=\alpha$ and $\lim \chi\left(y_{i}\right)=\beta \neq 0$ exists. In (7) set $y=y_{i}$ and take the limit. Because $\lim H\left(x, y_{i}\right)=0$ for every $x$, we get

$$
\chi(x) \cdot \alpha=\beta \cdot f(x)+h(x)
$$

with $h(x)=\lim _{i \rightarrow \infty} H\left(y_{i}, x\right)$. This function $h(x)$ is measurable and as a limit of continuous functions is continuous in at least one point [4,p. 164]. If $f$ vanishes at infinity, we have $\alpha=0$ and $f=-\beta^{-1} h$; consequently $f$ is continuous in at least one point and hence must be continuous everywhere. Therefore in this case $f \in \mathscr{C}_{\infty}$. If $f$ does not vanish at infinity we may choose $\alpha \neq 0$. Then (8) shows that $\chi$ must be measurable, hence continuous, and again $f=\beta^{-1}(\alpha \chi-h)$ is continuous. Then $g=\bar{\chi} f$ oscillates slowly at infinity and $f=\chi g$ has the form which the theorem requires.

To prove the sufficiency of the condition in Theorem 3, let $g$ be a bounded continuous function slowly oscillating at infinity. Then for every sequence $\left\{y_{n}\right\}$ tending to infinity,

$$
h_{n}(x)=g\left(x+y_{n}\right)-g\left(y_{n}\right)
$$

defines a sequence of uniformly bounded continuous functions with $\lim _{n \rightarrow \infty} h_{n}(x)=0$ for every $x$. Hence we have by Lebesgue's theorem for every bounded Radon measure $\mu: \lim \int h_{n}(x) d \mu(x)=0$ which means

$$
\left(T_{\mu} g\right)\left(y_{n}\right)-\mu(1) g\left(y_{n}\right) \rightarrow 0 .
$$

Therefore $T_{\mu} g-\mu(1) g$ is in $\mathscr{C}_{\infty}$, which means, that $g$ generates a minimal invariant subspace $(\tilde{g})$ in $\mathscr{B} / \mathscr{C}_{\infty}$ with

$$
T_{\mu} \tilde{g}=\mu(1) \tilde{g}=\hat{\mu}\left(\chi_{0}\right) \tilde{g}, \quad \chi_{0} \text { the unit-character. }
$$

If $\chi$ is a continuous character, then

$$
\begin{aligned}
\left(T_{\mu} \chi g\right)(x) & =\int \chi(x+y) g(x+y) d \mu(y)=\chi(x) \int g(x+y) d(\mu \cdot \bar{\chi})(y) \\
& \equiv \chi(x)(\mu \cdot \bar{\chi})^{\wedge}\left(\chi_{0}\right) g(x)\left(\bmod \mathscr{C}_{\infty}\right)=\hat{\mu}(\bar{\chi}) \chi g(x)\left(\bmod \mathscr{C}_{\infty}\right),
\end{aligned}
$$

i.e., we have $T_{\mu}(\chi g)^{\sim}=\hat{\mu}(\bar{\chi})(\chi g)^{\sim}$, where $(\chi g)^{\sim}=\chi g+\mathscr{C}_{\infty}$. Now a reference to (6) completes the proof of Theorem 3.

Finally we remark that Lemma (6) is trivial, if $G$ is separable; thus in this case the last paragraph already proves the theorem. 


\section{REFERENCES}

1. E. Hewitt, A survey of abstract harmonic analysis, Some aspects of analysis and probability; surveys in applied mathematics, Wiley, New York, 1958.

2. W. Rudin, Measure algebras on abelian groups, Bull. Amer. Math. Soc. 65 (1959), $227-247$.

3. E. Hewitt and S. Kakutani, A class of multiplicative linear functionals on the measure algebra of a locally compact abelian group, Illinois J. Math. 4 (1960), 553-574.

4. G. Aumann, Reelle Funktionen. Die Grundlehren der mathematischen Wissenschaften in Einzeldarstellungen mit besonderer Berücksichtigung der Anwendungsgebiete, Bd. 68, Springer, Berlin, 1954.

\section{UNIVERSITÄT HAMBURG,}

HAMBURG, Germany

TULANE UNIVERSTTY

New Orleans, Louisiana

\section{ERRATA TO VOLUME 104}

Joseph A. Wolf. Homogeneous manifolds of zero curvature, pp. 462-469.

Page 462 , line 13 of $\$ 2$. Delete the sentence “ $M_{s}^{n}$ is complete if it is homogeneous." For if $U$ is a nonzero totally isotropic linear subspace of $R_{s}^{n}$, then one can check that $R_{s}^{n}-U^{\perp}$ is homogeneous but not complete.

Add the hypothesis that $M_{s}^{n}$ is complete in Theorem 1 (page 466) and in Theorem 2 (page 467). 